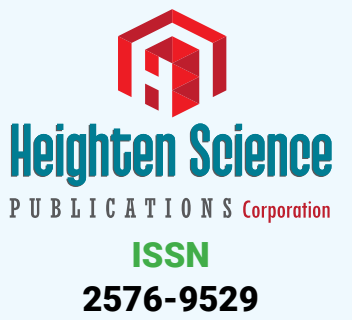

*Address for Correspondence: Kishore Kumar, Pakistan Institute of Medical Sciences, Islamabad, Pakistan,

Email: smciankishore@gmail.com

Submitted: 19 February 2019

Approved: 02 April 2019

Published: 03 April 2019

Copyright: () 2019 Kumar K, et al. This is an open access article distributed under the Creative Commons Attribution License, which permits unrestricted use, distribution, and reproduction in any medium, provided the original work is properly cited

Keywords: Chronic ambulatory peritoneal dialysis (Capd); Acute peritoneal dialysis (Apd); Peritonitis

Check for updates
Research Article

\section{Peritonitis: Culprit for peritoneal dialysis decline}

\author{
Kishore Kumar ${ }^{1 *}$, Jais Kumar ${ }^{2}$ and Chaudhri Naureen ${ }^{3}$ \\ 'Pakistan Institute of Medical Sciences, Islamabad, Pakistan \\ "Islamabad Medical and Dental College, Pakistan \\ ${ }^{3}$ Holy Family Hospital, Pakistan
}

\section{Abstract}

Peritonitis is the main complication of peritoneal dialysis caused the withdrawal of treatment like peritoneal dialysis which was used as primary treatment modality few years back in Pakistan. With this motto to know the exact cause of peritonitis this retrospective study was done and 35 out of 42 pervious peritoneal dialysis patients who had peritonitis were analyzed using old data. A total of 57 bags of all these peritonitis patients were analyzed in department of microbiology during the year 2007-2011. Out of these bags positive culture was obtained from 42 bags (74\%). Most of patients with positive culture were undergoing acute peritoneal dialysis $66.67 \%$ and rest were on chronic ambulatory peritoneal dialysis. Main concern was the yield of organisms causing culture positive peritonitis. It was found that bacterial peritonitis was positive in $80 \%$, fungal peritonitis was $11 \%$ and mycobacterium tuberculosis peritonitis was $09 \%$. Various culture techniques along with Gram Stain, Zeihl Nielsen Stain and Auramine stain were used for knowing the yield.

Limitations: Old and only small available data of peritonitis patients and stop of further peritoneal dialysis.

\section{Introduction}

Chronic renal disease is emerging a global disease problem. Unfortunately only limited treatment options are available for its treatments and their cost is another economic threat to most of the nations, especially in developing world like Asia and Africa [1]. Treatment options for chronic kidney disease includes renal replacement therapy in the form of dialysis or renal transplant. Transplant is not possible to get succeed in all and remaining dialysis is either hemodialysis or peritoneal dialysis. Now the main concern is about the supremacy of dialysis modality and when comes to economic burden, decreased hemodynamic instability and easy availability peritoneal dialysis overrides hemodialysis as primary mode of treatment. A decade before Peritoneal dialysis in the form of chronic ambulatory peritoneal dialysis (CAPD) or acute peritoneal dialysis (APD) was used as primary renal replacement therapy in Pakistan and all other developing world but later it was stopped suddenly and replaced by hemodialysis as primary renal replacement therapy [2-11]. But still most developing countries like Taiwan, Guatemala, etc. And some developed countries like china, Canada use it as primary RRT option due to les hemodynamic complications, more independency and of all very less economic Burdon on health care in these countries [8].

In Peritoneal dialysis peritoneum is used as filtration membrane and the Achilles heel of peritoneal dialysis is peritonitis which could be main factor for its stoppage in many countries. The mechanism of peritonitis is due to catheter related infection, contamination, transmigration, and haematogenous infection. Prompt diagnosis enables appropriate treatment and hence recovery and reduced morbidity and 
mortality. Collection of the sample bags, appropriate storage, and transfer to microbiology laboratory and culture techniques using a large quantity of drained dialysate increased the yield of positive cultures. As per recent ISPD guidelines less than $20 \%$ should be the culture negative peritonitis [7].

Mostly single pathogens were the cause of peritonitis. Multiple organisms were cultured in a small number of bags. Peritonitis can be relapsing, recurrent, refractory, and repeat. Peritonitis can result in the removal of P.D catheter especially in patients with refractory infections, associated with tunnel infection, bowel perforation and in fungal peritonitis.

With this ambition we looked for peritonitis positive patients in this retrograde study and tried to dig the different causes of this peritonitis. The aim of this retrospective study is to determine the prevalence of the culture positive and culture negative peritonitis and yield of organisms causing culture positive peritonitis during the period of 2007 to 2011 when peritoneal dialysis was common.

\section{Material and Methods}

A retrospective cohort study was conducted at the Pakistan Institute of Medical Sciences Islamabad. Data of all patients with P.D peritonitis from 2007-2011 was analyzed. Demographic profile of patients including age, gender, time and modality of Peritoneal Dialysis, number of peritonitis episodes, exit site or tunnel infection, culture positive or culture negative peritonitis and yield of organisms was collected.

The peritonitis episodes were analyzed in detail. P.D fluid was received as whole bag sample from each patient and complete analysis of P.D fluid was done by using, G-stain, Zeihl Nielsen Stain and Auramine Stain in department of Microbiology, Pakistan institute of medical sciences Islamabad.

The data was analyzed on SPSS 16 platform. Continuous variables like gender, sex, co-morbidities, choice of dialysis modality (CAPD or APD), culture positive or culture negative peritonitis, exit site or tunnel infection, type of organism were analyzed in percentage and numerical variables like age, peritonitis episodes, hemoglobin, albumin potassium levels, were analyzed as mean standard deviation.

\section{Results}

We analyzed 42 registered patients on peritoneal dialysis in Pakistan Institute of Medical Sciences between 2007-2011 and found that 35 (83.34\%) were diagnosed to have peritonitis.

Different base line characteristics obtained for these peritonitis positive patients and it was found that male were $26(74.1 \%)$ and female were $09(25.95 \%)$, The age, serum albumin and serum potassium were taken as mean standard deviation, and rest of characteristics were taken and continues variables. Among these patients $23(67.4 \%)$ were doing APD and 12(32.6\%) were doing CAPD. As shown in table 1.

Out of 35 peritonitis positive patients main site of infection was intraperetonal space in $26(74.07 \%)$ followed by tunnel and exit site infections as shown in table 2 .

The causal agents of peritonitis were Bacterial in 28(80\%), Fungal in $4(11 \%)$ and T.B in $3(9 \%)$ as shown in figure 1.

Total numbers of bags of all 35 peritonitis patients analyzed were 57 . Out of these bags culture positive bags were $42(74 \%)$ and negative were $15(26 \%)$ as shown in figure 2 .

Out of all 42 culture positive bags $7(16.67 \%)$ bags culture showed growth of more than one organism. The most common bacterial organism shown was coagulase negative staphylococcus (CoNS) in 8 bags, fungal organism was candida in 3 bags and MYCOBACTERIUM Tuberculosis in 3 bags and positivity of other organisms were shown in table 3. 
Table 1: Basic characterstics.

\begin{tabular}{|c|c|}
\hline GENDER & MALE 26 (74.1\%) \\
\hline Peritoneal Dialysis TYPE & FEMALE 09(25.9\%) \\
\hline & APD 23 (67.4\%) \\
\hline Diabetes Mallitus & CAPD 12(32.6\%) \\
\hline HTN & $18(33.5 \%)$ \\
\hline IHD & $35(64.8 \%)$ \\
\hline AGE & $22(40.7 \%)$ \\
\hline ALBUMIN & $56 \pm 15.07$ \\
\hline POTASIUM & $3.01 \pm 0.62$ \\
\hline
\end{tabular}

Table 2: Main sites of peritonitis in peritoneal dialysis.

\begin{tabular}{|c|c|}
\hline PERITONEAL CAVITY & $26(74.07 \%)$ \\
\hline TUNNEL INFECTION & $05(14.83 \%)$ \\
\hline EXIT SITE INFECTION & $04(11.4 \%)$ \\
\hline
\end{tabular}

Table 3: Different organisims of culture positive bags.

\begin{tabular}{|c|c|c|}
\hline \multirow{3}{*}{ CAUSE } & ORGANISM & NUMBER OF BAGS \\
\hline \multirow{3}{*}{ BACTERIAL } & CoNS & $08(19.05 \%)$ \\
\cline { 2 - 3 } & E.coli & $07(16.67 \%)$ \\
\cline { 2 - 3 } & Pseudomonas & $05(12 \%)$ \\
\cline { 2 - 3 } & Klebsiella pneumonia & $03(7.14 \%)$ \\
\cline { 2 - 3 } & Streptococcus & $03(7.14 \%)$ \\
\hline \multirow{2}{*}{ FUNGAL } & Others & $02(4.75)$ \\
\hline \multirow{2}{*}{ TB } & Candida & $03(7.14 \%)$ \\
\hline & Aspergilous/filamentous fungus & $01(2.39 \%)$ \\
\hline & Tuberculosis(T.B) & $03(7.14 \%)$ \\
\hline
\end{tabular}

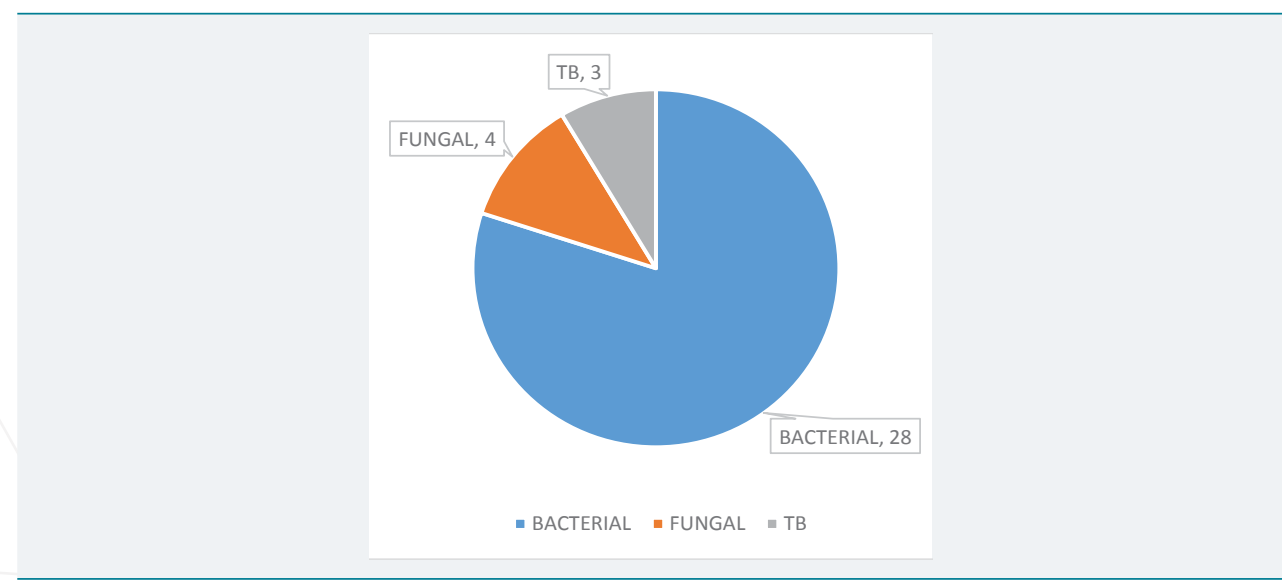

Figure 1: Causal Agents of Peritonitis.

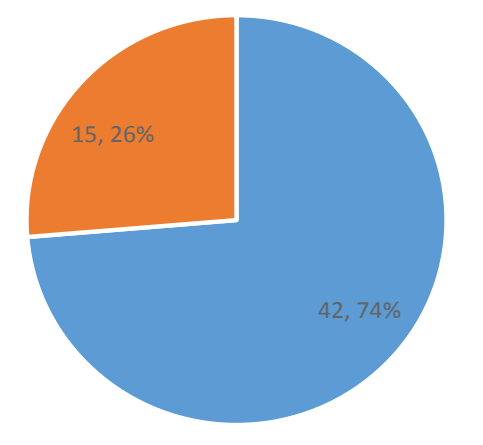

- CULTURE POSITIVE - CULTURE NEGATIVE

Figure 2: Culture positive and culture negative BAGS. 


\section{Discussion}

Peritonitis is common complication in peritoneal dialysis patients and one of major cause of patient's dropout from P.D. in recent years. To know the cause of peritoneal dialysis withdrawal from our center we have done this retrospective analysis of our previous dialysis patients who were using peritoneal dialysis as RRT. And we came to know that out of our 42 registered patients 35 had episode of peritonitis and some of them have repeated peritonitis.

As we know the yield of organism is very crucial for proper treatment and prevention from any infection and if the concern is to something special than its importance increased. With this in our mind we got help of our microbiology department and tried to dig out the causative factors in all those peritonitis positive patients. In the data of microbiology department a total of 97 bags of all 54 peritonitis positive patients were analyzed during that time and out of them $33 \%$ bags did not grew any organism and labeled as culture negative peritonitis.

With this we came to know that despite the improvement in diagnostic techniques still the main problem nephrologists would face is the presence of culture negative peritonitis. The causes of culture negative peritonitis could be due to prior antibiotic use, viral peritonitis, suboptimal culture techniques or some non-infectious causes of peritonitis.

In the remaining $74 \%$ of culture positive patients majority had bacterial cause followed by fungal and tuberculosis.

The treatment modality used was Intra Peritoneal (I.P) antibiotics and antifungal prophylaxis as per ISPD guidelines for peritonitis in all peritonitis patients except one patient who received Intra Venous (I.V) antibiotic treatment the most common regimen given was combination of I.P Cefazolin and Ceftazidime while changed later according to sensitivity.

Fungal and tuberculosis positive patients were the main cause of removal of P.D catheters in these patients in first episode and shifting from peritoneal dialysis to hemodialysis. Remainder withdrawn with time due to resistant to antibiotic treatment and increasing episodes culture negative peritonitis.

\section{Conclusion}

Peritonitis was found to be main cause of withdrawal from peritoneal dialysis from our center and almost all having peritonitis shifted to hemodialysis. Due to this frequency of infection our center stopped further taking patients in this program. But reason may vary center to center.

\section{Limitation}

Only limited and old data and no new induction of patients in P.D program since 2011 was main limitations.

\section{Recommendations}

With increase in expertise and limitation of complications peritoneal dialysis can be revived.

\section{References}

1. Rao M, Juneja R, Shirly RB, Jacob CK. Hemodialysis for ESRD in Southern India-a perspective from a tertiary referral care center. Nephrol Dial Transplant. 1998; 13: 2494-2500. Ref.: http://tinyurl. com/yyqnfk3p

2. Hussain R, Tufail M, Naqvi SAJ. Continuous ambulatory peritoneal dialysis as a model of renal replacement therapy in children and adults-Pakistan experience. Indian Journal of Peritoneal Dialysis. 2006; 11: 10-13. 
3. Abraham G, Mathew M, Padma G, Suresh S, Shroff S. Are three exchanges suitable for Asian patients on peritoneal dialysis? Perit Dial Int. 2003; 23 (Suppl 2): S45-47. Ref.: http://tinyurl.com/y6nu3w4f

4. Abraham G, Padma G, Matthew M, Shroff S. How to set up a peritoneal dialysis program: Indian experience. Perit Dial Int. 1999; 19 (Suppl 2): S184-186. Ref.: http://tinyurl.com/y69tmqyk

5. Samad MA, Iqbal S, Rashid HU. CAPD: experience in Bangladesh. Indian Journal of Peritoneal Dialysis. 2006; 10: 28-29. Ref.: http://tinyurl.com/y3yha8km

6. Dialysis Registry of Pakistan 2005-06. Karachi: The Kidney Foundation.

7. Kam-Tao Li P, Szeto CC, Piraino B, de Arteaga J, Fan S, et al. ISPD Peritonitis Recommendations: 2016 Update on Prevention and Treatment. Perit Dial Int. 2016; 36: 481-508. Ref.: http://tinyurl.com/y3lqcxlu

8. Liu FX, Gao X, Inglese G, Chuengsaman P, Pecoits-Filho R, et al. A Global Overview of the Impact of Peritoneal Dialysis First or Favored Policies: An Opinion. Perit Dial Int. 2015; 35: 406-420. Ref.: http://tinyurl.com/yxo53bzk

9. Ghali JR, Bannister KM, Brown FG, Rosman JB, Wiggins KJ, et al. Microbiology and outcomes of peritonitis in Australian Peritoneal dialysis patients. Perit Dial Int. 2011; 31: 651-662. Ref.: http://tinyurl.com/yytmzzj6

10. Pérez Fontan M, Rodríguez-Carmona A, García-Naveiro R, Rosales M, Villaverde $P$, et al. Peritonitisrelated mortality in patients undergoing Chronic peritoneal dialysis. Perit Dial Int. 2005; 25: 274-284. Ref.: http://tinyurl.com/y4wdjq3g

11. Davenport A. Peritonitis remains the major clinical complication of peritoneal dialysis: the London, UK, peritonitis audit 2002-2003. Perit Dial Int. 2009; 29: 297-302. Ref.: http://tinyurl.com/yyd4qb2o 